\title{
Hedtke, Reinhold
}

\section{Sozioökonomische Bildung}

Haushalt in Bildung \& Forschung 4 (2015) 3, S. 3-18

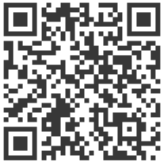

Quellenangabe/ Reference:

Hedtke, Reinhold: Sozioökonomische Bildung - In: Haushalt in Bildung \& Forschung 4 (2015) 3, S. 3-18

- URN: urn:nbn:de:0111-pedocs-203774 - DOI: 10.25656/01:20377

https://nbn-resolving.org/urn:nbn:de:0111-pedocs-203774

https://doi.org/10.25656/01:20377

in Kooperation mit / in cooperation with:

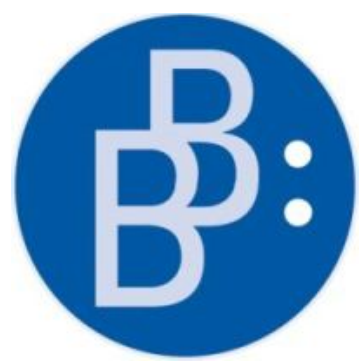

https://www.budrich.de

\section{Nutzungsbedingungen}

Gewährt wird ein nicht exklusives, nicht übertragbares, persönliches und beschränktes Recht auf Nutzung dieses Dokuments. Dieses Dokument ist ausschließlich für den persönlichen, nicht-kommerziellen Gebrauch bestimmt. Die Nutzung stellt keine Übertragung des Eigentumsrechts an diesem Dokument dar und gilt vorbehaltlich der folgenden Einschränkungen: Auf sämtlichen Kopien dieses Dokuments müssen alle Urheberrechtshinweise und sonstigen Hinweise auf gesetzlichen Schutz beibehalten werden. Sie dürfen dieses Dokument nicht in irgendeiner Weise abändern, noch dürfen Sie dieses Dokument für öffentliche oder kommerzielle Zwecke vervielfältigen, öffentlich ausstellen, aufführen, vertreiben oder anderweitig nutzen.

Mit der Verwendung dieses Dokuments erkennen Sie die Nutzungsbedingungen an.

\section{Terms of use}

We grant a non-exclusive, non-transferable, individual and limited right to using this document.

This document is solely intended for your personal, non-commercial use. Use of this document does not include any transfer of property rights and it is conditional to the following limitations: All of the copies of this documents must retain all copyright information and other information regarding legal protection. You are not allowed to alter this document in any way, to copy it for public or commercial purposes, to exhibit the document in public, to perform, distribute or otherwise use the document in public.

By using this particular document, you accept the above-stated conditions of use.

\section{Kontakt / Contact:}

\section{peDOcs}

DIPF | Leibniz-Institut für Bildungsforschung und Bildungsinformation Informationszentrum (IZ) Bildung

E-Mail:pedocs@dipf.de

Internet: www.pedocs.de

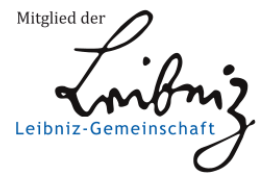




\section{Haushalt in}

Forschung

Sozioökonomische Allgemein-, Ernährungs- und Verbraucherbildung
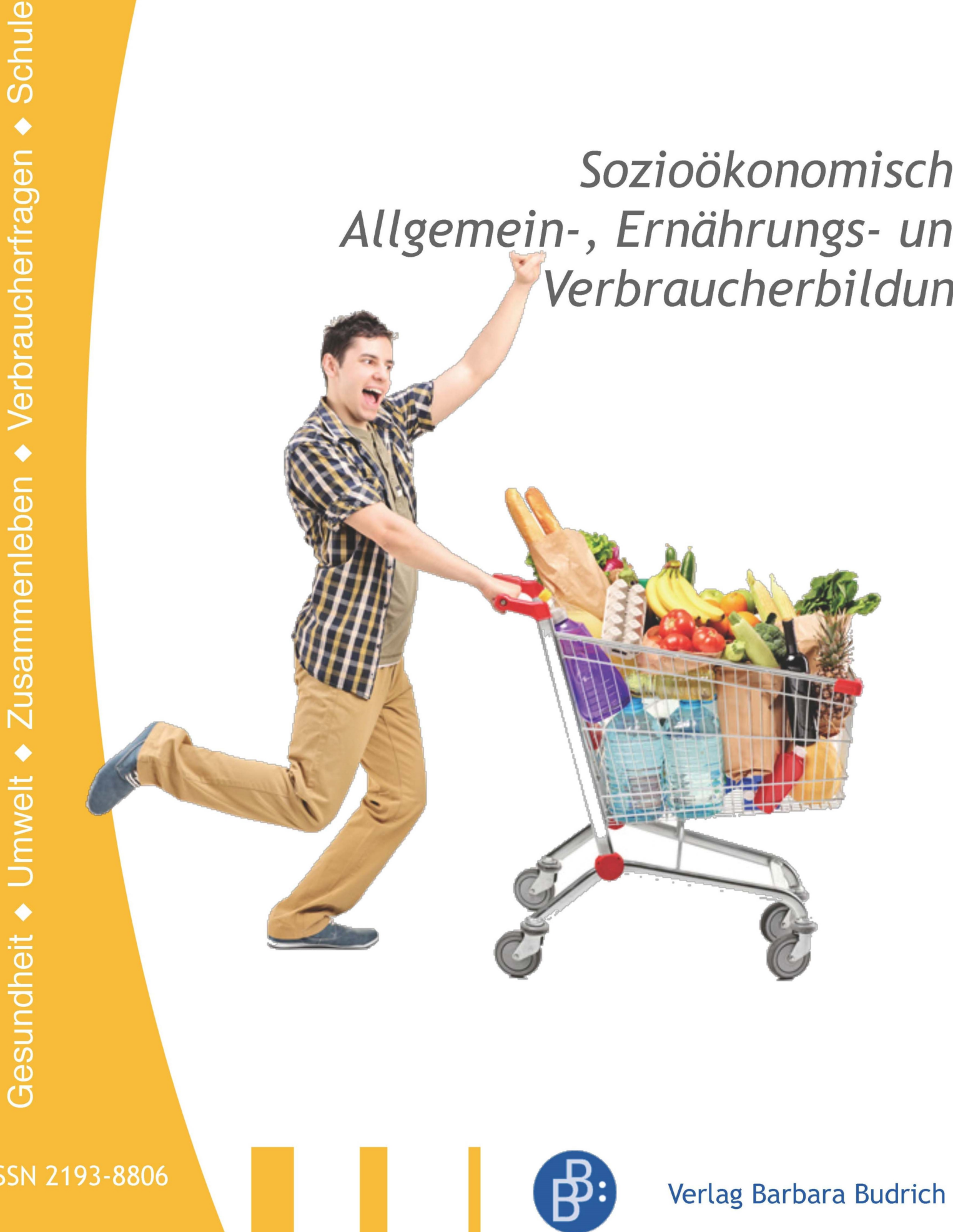
Inhaltsverzeichnis |

Werner Brandl

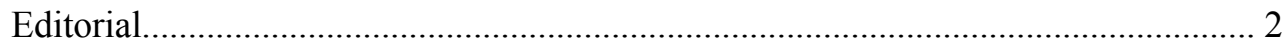

Reinhold Hedtke

Sozioökonomische Bildung................................................................................. 3

Angela Häußler

Fokus Haushalt - Überlegungen zu einer sozio-ökonomischen Fundierung der

Verbraucherbildung.

Uta Meier-Gräwe

Caring. Cooking. Cleaning. Warum wir die Arbeit des Alltags gesellschaftlich neu und geschlechtergerecht verteilen müssen.

Jana Rückert-John

Der Wandel des Ernährungsalltags als Herausforderung für die Ernährungs- und

Verbraucherkommunikation

Carolin Kölzer \& Volker Schwier

Sozioökonomische Bildung im Sachunterricht der Grundschule - mehr

als ,work, earn, play“.

Stefanie Nolte

Mit reflektierten Konsumentscheidungen die Zukunft fairändern - eine

Zukunftswerkstatt.

Uwe Becker

Alter, Armut, Schulden. Warum Prävention ein Euphemismus ist.

Werner Brandl

Reinhold Hedtke (Hrsg.). Was ist und wozu Sozioökonomie?.

Angela Häußler \& Barbara Methfessel

Uta Meier-Gräwe (Hrsg.). Die Arbeit des Alltags. Gesellschaftliche Organisation

und Umverteilung.

Anna Maria Hoff

Jana Rückert-John (Hrsg.). Soziale Innovation und Nachhaltigkeit.

Perspektiven sozialen Wandels.

HaBiFo-Fachtagung und Preis 2016. 
Sozioökonomische Bildung |

\section{Reinhold Hedtke}

\section{Sozioökonomische Bildung}

Sozioökonomische Bildung steht in einer langen Tradition. Das trifft auch auf die Konflikte um die Gestalt der allgemeinen ökonomischen Bildung zu. Der Beitrag greift die Tradition auf und begründet Grundlinien einer modernen sozioökonomischen Bildung. Sie macht ,Wirtschaft und Wirtschaften in der Gesellschaft` zum Gegenstandsbereich von Bildung und Lernen und bearbeitet ihn mit Wissen und Verfahren aus den Sozialwissenschaften.

Schlüsselwörter: Wirtschaftsdidaktik, Sozialwissenschaften, Bildungskonzeption, Domäne

Seit fünf Jahren hält die jüngste fach- und bildungspolitische Debatte um die schulische Form der ökonomischen Bildung an. Die vehemente Forderung von Wirtschaftsverbänden und Teilen der Wirtschaftsdidaktik nach einem separaten Schulfach Wirtschaft hat massive Kritik hervorgerufen. Die Kritikerinnen lehnen ein rein wirtschaftswissenschaftliches Fach ab und berufen sich dabei auf die Konzeption sozioökonomischer Bildung. Anders als es vor diesem Hintergrund scheinen mag, hat die sozioökonomische Bildung in Deutschland und Europa eine lange Tradition. Diese Tradition und ihre Aktualität ruft der Beitrag zunächst kurz in Erinnerung (1.). In einem zweiten Schritt verortet er die sozioökonomische Bildung in Bezug auf ihren Gegenstandsbereich (2.1), ihr Bildungsverständnis (2.2), ihren Wissenschaftsbezug (2.3) und ihren Ort in der gesellschaftlichen Domäne (2.4). Drittens gibt der Text einen zusammenfassenden Überblick über wesentliche Unterschiede zwischen sozioökonomischer und konventioneller ökonomischer Bildung (3.).

\section{Eine innovative Tradition}

Seit Jahrzehnten gibt es latente und manifeste Konflikte um die Frage, wie man wirtschaftliche Inhalte und Themen in der Stundentafel und im Curriculum verankern soll. Dabei hat sich in Deutschland und Europa eine Vielfalt unterschiedlicher Lösungen entwickelt und etabliert. Auch die aktuellen Auseinandersetzungen um ein Schulfach Wirtschaft kann man nur dann verstehen, wenn man sie in den traditionellen Konfliktlinien verortet. Einerseits herrscht seit den 1960er Jahren Einigkeit darüber, dass das Themenfeld Wirtschaft curricular zu verankern ist. Andererseits sind die Inhalte und die Form, in der dies organisiert werden soll, seit jeher umstritten. 


\section{Sozioökonomische Bildung}

\subsection{Die ökonomistische Intervention}

Die fachdidaktischen Kontroversen um ökonomische oder sozioökonomische Bildung reichen weit zurück und wurzeln in den 1960er Jahren. Sie drehen sich seit jeher um die Fragen der Schulfachkonstruktion (Separatfach oder Ankerfach), der Auswahl der Bezugsdisziplinen (Wirtschaftswissenschaften oder Sozialwissenschaften), der Priorität von Paradigmen (Monismus oder Pluralität), der Leitideen (Fachwissenschaftsorientierung oder Situationsorientierung) und das politischpädagogische Programm (Anpassung oder Kritik).

Tab. 1: Vom sozioökonomischen Konsens zur ökonomistischen Kehrtwende? ${ }^{1}$

\begin{tabular}{|c|c|c|}
\hline & $\begin{array}{l}\text { Der bildungspolitische } \\
\text { Konsens von 2000: } \\
\text { Sozioökonomische Bildung }\end{array}$ & $\begin{array}{l}\text { Die fachpolitische } \\
\text { Wende von 2010: } \\
\text { Ökonomistische Bildung }\end{array}$ \\
\hline Lernbereich & $\begin{array}{l}\text { Lernbereich Sozioökonomie } \\
\text { (Arbeits- und Wirtschaftswelt) }\end{array}$ & $\begin{array}{l}\text { Domäne Ökonomie } \\
\text { (Wirtschaftswissenschaft) }\end{array}$ \\
\hline Fach & Schulfach Wirtschaft & Schulfach Ökonomie \\
\hline Disziplinbezug & $\begin{array}{l}\text { interdisziplinär, } \\
\text { fächerübergreifend }\end{array}$ & monodisziplinär, fachsepariert \\
\hline Profil & praxisorientiert & k. A. \\
\hline Objektbereich & $\begin{array}{l}\text { Erwerbsarbeit und } \\
\text { Beschäftigungssystem, } \\
\text { Wirtschafts- und } \\
\text { Gesellschaftssystem, Politik }\end{array}$ & $\begin{array}{l}\text { universal, kein abgrenzbarer } \\
\text { Gegenstandsbereich }\end{array}$ \\
\hline Bildungsperspektive & $\begin{array}{l}\text { ökonomische, soziale, } \\
\text { politische, rechtliche, } \\
\text { ökologische und technische } \\
\text { Zusammenhänge, aktuelle } \\
\text { wirtschaftliche und politische } \\
\text { Probleme }\end{array}$ & $\begin{array}{l}\text { Perspektive „des“ Ökonomen: } \\
\text { Verbesserung der } \\
\text { (wirtschaftlichen) Situation; } \\
\text { Beurteilungsmaßstab: } \\
\text { Effizienz. }\end{array}$ \\
\hline Lehrerausbildung & $\begin{array}{l}\text { interdisziplinäres, } \\
\text { lehramtsbezogenes Studium } \\
\text { der Wirtschafts- und } \\
\text { Sozialwissenschaften }\end{array}$ & $\begin{array}{l}\text { Studium der Betriebs- und } \\
\text { Volkswirtschaftslehre, ergänzt } \\
\text { um Wirtschaftsprivatrecht }\end{array}$ \\
\hline Koalition & $\begin{array}{l}\text { Deutscher Elternverein, } \\
\text { Realschullehrerverband, } \\
\text { DeGöB, BDA, DGB; } \\
\text { Bundeselternrat (ohne Votum } \\
\text { zum Fach) }\end{array}$ & $\begin{array}{l}\text { Gemeinschaftsausschuss der } \\
\text { deutschen gewerblichen } \\
\text { Wirtschaft (Arbeitgeber- und } \\
\text { Branchendachverbände), vier } \\
\text { Wirtschaftsdidaktiker }^{2}\end{array}$ \\
\hline
\end{tabular}




\section{Sozioökonomische Bildung}

Eine lange Tradition haben auch die bildungspolitischen Konfliktlinien. Im Übergang von den 1970er zu den 1980er Jahren vertrat der DGB in den Auseinandersetzungen um die Ausgestaltung der Arbeitslehre eine interdisziplinär-integrierte Konzeption ökonomischer Bildung, während die Arbeitgeberverbände einen fachwissenschaftlich-fachsystematischen Ansatz bevorzugten. Einen anhaltenden, derzeit latenten Konflikt erzeugt auch die Frage, ob der Fachzugriff dem Leitkonzept Arbeit oder Wirtschaft(swissenschaft) folgen soll.

Als bildungspolitisch besonders bemerkenswert erscheinen die Jahre 2000 und 2010. Arbeitgeber und Gewerkschaften formulierten im Jahr 2000 einen relativ breit unterstützten Konsens über eine sozialwissenschaftlich orientierte, sozioökonomische Bildung (Gemeinsame Initiative, 2000). Die Arbeitgeber- und Wirtschaftsverbände kündigten ihn zehn Jahre später durch ein von ihnen beauftragtes Fachgutachten faktisch auf (Retzmann et al., 2010). Sie vollzogen eine fachpolitische Wende, die sich schlüssig in die Strategie für ein separates Schulfach Wirtschaft einfügt (Hedtke, 2012).

Deutschland steht mit dieser Konfliktlage nicht allein. Etwa zeitgleich organisieren Wirtschaftsverbände und konservativ-liberale Politiker in einer Reihe von europäischen Ländern ähnliche Vorstöße (vgl. Hedtke, 2015a).

Wie weit sich die Wirtschaftsverbände und Teile der Wirtschaftsdidaktik mit ihrem neuen Ansatz von der sozioökonomischen Konsensposition entfernt haben, zeigt die Gegenüberstellung der wesentlichen Merkmale des sozioökonomischen und des ökonomistischen Konzepts (vgl. Tabelle 1).

\subsection{Die sozioökonomische Tradition}

Die fachdidaktischen, curricularen und bildungspolitischen Wurzeln der sozioökonomischen Bildung lassen sich weit zurückverfolgen. Die Didaktik sozioökonomischer Bildung an allgemeinbildenden Schulen stützt sich in Deutschland auf eine über fünfzigjährige Tradition (vgl. zum Folgenden Hedtke, 2015c). Bereits Mitte der 1960er Jahre entwickelte Hans Bokelmann sein Konzept ökonomisch-sozialethischer Bildung. Die Traditionslinie sozialwissenschaftlich-ökonomischer Bildung wurzelt in den 1970er Jahren. Sie spiegelt den damaligen Relevanzgewinn der Sozialwissenschaften in der Gesellschaft und im Bildungssystem.

Mit dem sozialwissenschaftlich lebenssituationsorientierten Ansatz setzen Bodo Steinmann und Dietmar Ochs diese Linie in den 1990er Jahren fort. Zugleich ergänzen sie die Sozialwissenschaftsorientierung um Orientierung an Handlungskompetenz einerseits, Förderung von individueller und gesellschaftlicher Emanzipation andererseits. Programmatisch fordern sie, „die ökonomische Bildung zu einer umfassenden Gesellschaftslehre auszugestalten“ und sie „in ein auf die Gesellschaft als Ganzes bezogenes Curriculum“ einzubetten (Steinmann, 1997, S. 20). Etwa zeitgleich startet eine Serie von engagierten Plädoyers für sozioökonomische Bildung, 


\section{Sozioökonomische Bildung}

vorgetragen vor allem von Dietmar Kahsnitz, Günther Seeber und Peter Weinbrenner (vgl. z. B. Kahsnitz, 1999, Seeber, 1997).

Die damals vorgelegten fachdidaktischen Konzepte enthalten bis heute geltende Grundelemente sozioökonomischer Bildung. Dazu zählen insbesondere das sozialwissenschaftliche Selbstverständnis, die Selbstverpflichtung auf eine kritische Bildung, die ihre Kritik auch auf die Wirtschaftsgesellschaft und den Ökonomismus der orthodoxen Wirtschaftswissenschaften richtet, die pragmatische Orientierung an realweltlichen Problemen und Lebenssituationen, die multidisziplinäre Analysen und Lösungsvorschläge verlangen, sowie die dezidierte Ausrichtung auf Persönlichkeitsbildung.

Auch im schulisch-curricularen Kontext geht die sozioökonomische Bildung auf eine lange Tradition zurück. Dort dominiert - neben der bereits erwähnten arbeitsorientierten Traditionslinie (z. B. Arbeitslehre, Arbeit-Wirtschaft-Technik) - die sozialwissenschaftliche Strömung ökonomischer Bildung (vgl. Hedtke, 2015c). Sie manifestiert sich heute in der Stundentafelstruktur und den Fachphilosophien vieler europäischer Länder mit Fächern wie Gesellschaft-Wirtschaft-Politik, Sozialwissenschaften, Politik/Wirtschaft oder Sciences Économiques et Sociales.

Zugleich zeigt sich im europäischen Vergleich eine große Vielfalt der Fächertraditionen: Weitere Strömungen verankern den Gegenstandsbereich Wirtschaft und ökonomische Kompetenzen in humanwissenschaftlichen oder in hauswirtschaftlichkonsumorientierten Integrationsfächern. Bis auf wenige Ausnahmen organisieren aber die meisten der einschlägigen Schulfächer der Sekundarstufe I die ökonomische Bildung in einer multi- oder transdisziplinären Form.

Schließlich kommen in Stundentafeln und Fächerkonstrukten auch bildungspolitische Überzeugungen über angemessene Formen ökonomischer Bildung zum Ausdruck. Ein starkes inhaltliches Motiv war und ist, Wirtschaft, Gesellschaft und Politik im wechselseitigen Zusammenhang zu thematisieren.

\subsection{Zur Zukunft der sozioökonomischen Bildung}

Die Auseinandersetzungen um das 2010 vorgestellte ökonomistische Bildungskonzept bescherten der sozioökonomischen Strömung in der Wirtschaftsdidaktik wieder vermehrte Aufmerksamkeit und motivierten dazu sie weiterzuentwickeln. Die konzeptionelle Debatte führte zu einer Fachtagung im Jahr 2012, ein Herausgeberband zur sozioökonomischen Bildung folgte (Fischer \& Zurstrassen, 2014). Inzwischen liegen auch konkrete Vorschläge für eine sozioökonomische Unterrichtspraxis vor (Bundeszentrale für politische Bildung, 2014).

Die Sozioökonomiedidaktik wird sich als fachdidaktische Forschungstradition konsolidieren. Dazu wird sie zum einen ihre konzeptionellen Grundlagen ausdifferenzieren und insbesondere empirische und theoretische, sozialwissenschaftliche, allgemeindidaktische und bildungstheoretische Anschlüsse ausarbeiten. Zum anderen wird sie den „Kanon“ bildungsrelevanter Wissensbestände und Kompetenzen, wie er 


\section{Sozioökonomische Bildung}

sich etwa in Standards und Kernlehrplänen ausdrückt, auf den aktuellen Stand des sozialwissenschaftlichen Wissens über Wirtschaft und Wirtschaften bringen. Auf dieser Basis nimmt sie die Modernisierung der auf Wirtschaft bezogenen Kerncurricula an allgemein bildenden Schulen in Angriff. Dabei wird sie die allgegenwärtigen Ansprüche auf alltagsweltlich-reale Handlungsrelevanz von wissenschaftlichen Wissensbeständen und Verfahrensweisen kritisch prüfen.

\section{Wie verortet sich die sozioökonomische Bildung?}

Die Konzeption der sozioökonomischen Bildung gründet auf vier Grundsatzentscheidungen. Sie betreffen den Gegenstandsbereich, das Bildungsverständnis, die Bezugswissenschaften und die Domäne, der sie angehört.

\subsection{Wirtschaft in der Gesellschaft}

In allgemeinster Formulierung begreift die Sozioökonomiedidaktik Wirtschaft und Wirtschaften in der Gesellschaft als den genuinen Gegenstandsbereich von sozioökonomischer Bildung. Im Mittelpunkt steht der gesellschaftliche Realitätsbereich Wirtschaft - differenzierungstheoretisch gesprochen: das Funktionssystem Wirtschaft - samt Produktion, Konsum und Verteilung als seinen Beiträgen zur Sicherung der materiellen Reproduktion der Gesellschaft und ihrer Mitglieder.

Neben diesen Reproduktionsfunktionen geht es um seine Institutionen, etwa Privateigentum und Geld, Freihandel und Governance, wirtschaftliche und wirtschaftlich relevante Regeln, Kulturen oder Normen, sowie seine Koordinationsformen und Konventionen. Beispiele sind Märkte und Netzwerke, Organisationstypen wie Unternehmen oder Verbände, handwerkliche oder industrielle, ökologische oder regionale Produktionsformen, usw. Selbstverständlich gehören das wirtschaftliche Denken, Verhalten und Handeln der individuellen und kollektiven Akteure dazu.

Die Sozioökonomiedidaktik greift Grundgedanken der Konventionenökonomie auf und betrachtet ihren Gegenstandsbereich und seine Institutionen als fundamental pluralistisch, die wirtschaftliche Praxis als strukturell plural und deshalb für die Akteure interpretations-, koordinierungs- und verständigungsbedürftig (vgl. z. B. Eymard-Duvernay, Favereau, Orléan, Salais \& Thévenot, 2011, Diaz-Bone, 2015). Damit sind Wirtschaft und Wirtschaften als vielfältige gesellschaftliche, historische, kulturelle und wandelbare Phänomene zu behandeln. Diese Vielfalt schließt auch die ökonomistische, auf Verbesserung/Wachstum und Wirtschaftlichkeit/Effizienz fokussierte Perspektive ein, aber nicht als einzige, sondern als eine Sichtweise neben anderen.

Sozioökonomiedidaktik konturiert und konstruiert die „Sache“, um die es ihr bei Lernen und Bildung geht, also durch einen primär gegenstandsorientierten Zugriff. Das gegenstandsbezogene Selbstverständnis teilt sie mit ihren fachdidaktischen 


\section{Sozioökonomische Bildung}

Nachbardisziplinen und mit den Schulfächern in der sozialwissenschaftlichen Domäne, insbesondere mit der Politikdidaktik und den diversen Fächern der politischen Bildung. Auch Geographiedidaktik und Geschichtsdidaktik identifizieren sich über ihren spezifischen Gegenstandsbereich: Natur und Gesellschaft in Räumen bzw. gesellschaftliches Geschichtsbewusstsein und geschichtskulturelle Lebenswelt. In diesem fachdidaktischen und schulfachlichen Umfeld sieht sich die sozioökonomische Bildung gut aufgehoben.

Ihrem Gegenstand nähert sich die sozioökonomische Bildung selbstverständlich vor allem mit sozialwissenschaftlichen Konzepten - das ist eine triviale Verbindung von Gegenstandsorientierung und Wissenschaftsorientierung. Die Subjekte selbst sind durch ihr individuelles Erleben und Erleiden und durch kollektive Erzählungen, Erfahrungen, Erwartungen und Erklärungsmuster vielfach in das wirtschaftliche Feld verstrickt. Ihre wirtschaftlichen Lagen, Routinen, Denkweisen und Entscheidungen sind umfassend in soziale und kollektive Kontexte eingelagert, man denke an private Haushalte, Familien und Peergroups, Unternehmen und Arbeitsbeziehungen, Konsum-, Anlage-, Arbeits- und Lebensstile. Deshalb muss man den sozialwissenschaftlichen Zugriff auf den Gegenstandsbereich und subjektorientierte Zugänge zu Wirtschaft sozioökonomiedidaktisch miteinander verknüpfen.

\subsection{Beitrag zur gesellschaftlichen Bildung}

Wer von sozioökonomischer Bildung spricht, muss sie von sozioökonomischem Lernen unterscheiden. Bildungsprozesse kann man als längerfristiger, integrativer, komplexer, tiefergehend und weiterreichend auszeichnen als Lernprozesse. Während es beim Lernen um das Finden adäquater Antworten auf Probleme geht, die sich den Subjekten innerhalb eines gegebenen Rahmens stellen, transformiert Bildung den Rahmen des Lernens (vgl. z. B. Kokemohr, 2007). Lernen verändert und erweitert die verfügbaren Möglichkeiten des Denkens und Handelns. Die Subjekte eignen sich also neues Wissen und Können an, sie verknüpfen es mit dem bisherigen und schreiben dieses modifiziert und erweitert fort. Bildung dagegen verändert, mehr oder weniger stark und dauerhaft, die grundlegenden Verhältnisse, die das Subjekt zu sich selbst, zu anderen und zur Welt eingeht und entwickelt (Selbst-, Sozial-, Weltverhältnisse; vgl. z. B. Koller, 2007).

Bildung basiert auf Lernen. Bildungsprozesse heben sich aber in der Reichweite ihrer Veränderungswirkungen von Lernprozessen ab. Es handelt sich also um eine graduelle Unterscheidung mit fließenden Übergängen, nicht um eine Dichotomie Bildung/Lernen.

Als Bildungsprozess bezeichne ich im schulischen Kontext und für die sozioökonomische Bildung nicht nur und nicht in erster Linie tief greifende Transformationen. Vielmehr verwende ich den Bildungsbegriff auch für Veränderungen von einzelnen politischen, ökonomischen oder gesellschaftlichen Einstellungen, Werthaltungen, Erklärungs- und Deutungsmustern, Sinngebungen und Praktiken, 


\section{Sozioökonomische Bildung}

Identifikationen oder Zugehörigkeiten einer Person. Die Beispiele zeigen, worauf es bei der Unterscheidung ankommt: Bildung verändert auch die Person und ihre Verhältnisse, nicht nur ihr Wissen und Können.

Sozioökonomiedidaktik geht dabei davon aus, dass Personen heute typischerweise mehrere Identitäten haben und sich mit ihnen in einer Mehrzahl von heterogenen und widersprüchlichen Wirtschaftswelten bewegen (Pluralismus der Person, Thévenot, 2011). Unter Bedingungen umfassender Pluralität geraten individuelle und kollektive wirtschaftliche Entscheidungen und Handlungen zunehmend unter Rechtfertigungsdruck. Sie werden aus unterschiedlichen Perspektiven heraus kritisiert. Arbeitnehmerrechte, soziale Gerechtigkeit, Umweltverträglichkeit, fairer Handeln, Nachhaltigkeit und Tierrechte sind nur einige Beispiele dafür. Deshalb verliert der Begründungstyp monetäre Effizienz stark an Überzeugungskraft.

Im Gegenstandsbereich Wirtschaft treffen Jugendliche typischerweise auf bildungsrelevante Themen- und Erfahrungsfelder, in die sie als Person involviert sind und die sie mit pluralen Welten und Werten konfrontieren, etwa Geld, Konsum, Arbeit und Beruf, Wirtschafts- und Sozialpolitik. Insbesondere an Erwerbsarbeit und Konsum machen viele ihre multiplen Identitäten fest.

Das auf diese Felder bezogene Denken und Handeln der Lernenden ist gesellschaftlich geprägt, seine Kontexte sind kollektiv definiert oder organisiert. Die kollektiven Kontexte bilden einen pluralen und keinen einheitlichen Horizont. Das illustrieren beispielweise die Lebensstile oder die Arbeitseinstellungen, die vielfältig, aber nicht beliebig sind.

Sozioökonomiedidaktik berücksichtigt deshalb, dass die persönlichen, bildenden Veränderungen oft auf kollektive Kontexte und Kommunikationen Bezug nehmen. Auch die (sozioökonomische) Bildung selbst vollzieht sich - zumindest auch - als ein sozialer Prozess. Das trifft besonders dann zu, wenn sich Bildung im Umfeld von schulisch, curricular und gruppenförmig organisierten Settings entwickelt. Das triviale Exempel dafür ist die Schulklasse.

Aus diesen - und weiteren Gründen - ist sozioökonomische Bildung eine angemessene Zugangsweise und ein wesentlicher Beitrag zur gesellschaftlichen Bildung von Kindern und Jugendlichen.

\subsection{Bezug zu den Sozialwissenschaften}

Sozioökonomische Bildung versteht sich als Teil einer umfassenderen sozialwissenschaftlich fundierten gesellschaftlichen Bildung. Sie stellt sich damit in die sozialwissenschaftliche Tradition der Fachdidaktiken. Aus der skizzierten Gegenstandsorientierung und aus dem Prinzip der Wissenschaftsorientierung ergibt sich ein multidisziplinärer Wissenschaftsbezug der sozioökonomischen Bildung.

In den Sekundarstufen I und II folgen Bildung und Lernen den allgemeineren und grundlegenden Prinzipien Wissenschaftsorientierung oder Wissenschaftspropädeutik, nicht Disziplinorientierung oder Disziplinpropädeutik. Die Sozioökono- 


\section{Sozioökonomische Bildung}

miedidaktik schöpft den wissenschaftlichen Teil ihrer bildungsrelevanten Inhalte und Verfahren aus einer Mehrzahl von sozialwissenschaftlichen Bezugsdisziplinen. Auch die politische, die geographische und die historische Bildung betrachten ihre bezugswissenschaftliche Interdisziplinarität als ein konstitutives Charakteristikum.

Soweit die Sozioökonomiedidaktik relevante Probleme als Auswahlkriterien dafür wählt, entspricht ihr Vorgehen den problemorientierten Forschungstraditionen, die man etwa in der Betriebswirtschaftslehre, der Managementlehre, der Personalwirtschaft, der Konsumforschung oder der Marketinglehre findet.

Die sozialwissenschaftlichen Disziplinen überschneiden sich in ihren Methodologien, Fragestellungen, Theorien und Methoden. Wichtige Beispiele sind Konzepte wie Zweckrationalität und Rationalhandlung, Institutionen und Organisationen, Globalisierung und Ökonomisierung, Gemeinwohl und Interesse oder Wettbewerb und Macht. Konsequenterweise versteht sich etwa die Konventionenökonomie als transdisziplinär-integrativer Ansatz (Diaz-Bone, 2015).

Zugleich ist jede der sozialwissenschaftlichen Disziplinen Politikwissenschaft, Volkswirtschaftslehre, Betriebswirtschaftslehre und Soziologie disziplinintern multiparadigmatisch strukturiert und weder theoretisch noch methodisch homogen (Kornmesser \& Schurz, 2014). Dass mehrere theoretische Grundkonzepte existieren, ist unmittelbar praxisrelevant, da Theorien implizite oder explizite Handlungsempfehlungen enthalten, sei es für Unternehmen oder politische Instanzen. Der wissenschaftliche und paradigmatische Pluralismus und seine praktisch-politischen Folgen werden nicht nur in der Volkswirtschaftslehre, sondern auch in der Betriebswirtschaftslehre und im Rechnungswesen breit diskutiert (z. B. Davis, 2014, Schanz, 2014, van der Meer-Kooistra \& Vosselman, 2012).

Disziplinäre Differenzen zwischen den Sozialwissenschaften mögen im Wissenschaftssystem wichtig sein. Für die schulische Bildung haben sie angesichts der Aufgabe, die gesellschaftliche (politische, wirtschaftliche, soziale) Welt elementar und Grund legend zu erschließen, nur nachrangige Bedeutung.

Deshalb befragt die Sozioökonomiedidaktik sowohl die Sozialwissenschaften (transdisziplinäres Wissen) als auch ihre Einzelwissenschaften (disziplinäres Wissen) nach bedeutsamen Beiträgen zur sozioökonomischen Bildung von Kindern und Jugendlichen. Nach fachdidaktischen Kriterien wählt sie aus ihnen einige wenige, besonders bildungsrelevante Wissensbestände und Verfahrensweisen aus. Eine systematische Einführung in einzelne sozialwissenschaftliche Disziplinen gehört dagegen nicht zu den Aufgaben der Schule. Sozioökonomische Bildung ist deshalb sozialwissenschaftlich transdisziplinär orientiert.

$\mathrm{Zu}$ den Disziplinen und Forschungstraditionen, deren Denkweisen und Wissensbestände die Sozioökonomiedidaktik als besonders bildungsträchtig betrachtet zählen: Volkswirtschaftslehre, Sozioökonomie, Konventionenökonomie, Wirtschafts-, Arbeits- und Konsumsoziologie sowie die Politische Ökonomie. Die Sozioökonomiedidaktik bezieht sich dabei jeweils auf die internationale Forschungsgestalt der 


\section{Sozioökonomische Bildung}

Disziplinen. Damit rücken die in der konventionellen Wirtschaftsdidaktik vorherrschenden, national verengten Versionen und Konzeptionen, etwa der deutsche Ordoliberalismus oder die deutsche soziale Marktwirtschaft, in den Hintergrund.

Man würde den Lernenden wesentliches wirtschaftliches Wissen und Können vorenthalten, würde man ihnen für die folgenden Fragen nur oder überwiegend Wissen aus den Wirtschaftswissenschaften anbieten: Was ist Geld? Wie funktionieren Geldwirtschaften? Was bedeutet Arbeit, wie wird sie organisiert und was folgt daraus? Was sind Märkte, wie entstehen, bestehen, funktionieren und was bewirken sie? Wie hängen Konkurrenz und Kooperation zusammen? Was sind und wie funktionieren Unternehmen, was setzen sie voraus, was bringen sie hervor? Was ist und wie berechnet man Gewinn? Wer hat warum wirtschaftliche Macht und wie wirkt sie? Was kennzeichnet Marktwirtschaften und was unterscheidet sie untereinander? Kann und soll man sie politisch steuern? Welche alternativen Konzepte von Marktwirtschaft und welche Alternativen zu ihr gibt es? Wer trägt wirtschaftliche Risiken und wer bleibt davon verschont? Wie funktioniert und wem nützt die kapitalgedeckte Altersvorsorge? Woher kommen Innovationen? Wie entsteht Wachstum? Was heißt wirtschaftliches Handeln und an welchen Regeln und Normen orientiert es sich? Was sind Institutionen, wie entstehen sie und wie verändern sie sich? Usw.

Auf Wirtschaftswissenschaften verengte Lehrpläne behindern die ökonomische und verhindern die sozioökonomische Bildung. Sie schließen das reichhaltige relevante Wissen über Wirtschaft aus den anderen Sozialwissenschaften von vornherein und willkürlich aus. Es findet faktisch auch in anderen Domänen oder an anderen Stellen im Curriculum keinen Platz. Diese disziplinär motivierte Ausgrenzung verstößt deshalb gegen das Prinzip der Wissenschaftsorientierung.

Selbstverständlich gibt es Inhaltsfelder, bei denen volkswirtschaftliches Wissen gegenüber dem aus anderen Sozialwissenschaften in der sozioökonomischen Bildung einen Vorrang genießt. Sie stammen vor allem aus dem Bereich der Makroökonomie (in dieser Domäne kann man noch am ehesten ein Spezifikum der Volkswirtschaftslehre gegenüber anderen Sozialwissenschaften sehen). Dazu zählen etwa Beschäftigung, Konjunktur, Investition, Preisniveau, Währung, Gleichgewicht oder Außenwirtschaft. Auch die makroökonomischen Konzepte sind wissenschaftlich kontrovers.

Insgesamt muss die Sozioökonomiedidaktik systematisch herausarbeiten, welche sozialwissenschaftlichen oder disziplinären Konzepte im Zusammenhang mit welchem der curricular kanonischen Inhaltsfelder erworben, angewendet und dann mit anderen Konzepten verglichen werden sollen.

\subsection{Subdomäne Wirtschaft in der Domäne Gesellschaft}

Sozioökonomische Bildung verortet sich in der Domäne Gesellschaft, die im engeren Sinne die Subdomänen Politik, Wirtschaft und Gesellschaft umfasst. Die Domäne und ihre Subdomänen bezeichnen im Sinne von Weinert Inhaltsgebiete oder Wis- 


\section{Sozioökonomische Bildung}

sensfelder, sie sind nicht identisch mit den Disziplinen Politikwissenschaft, Wirtschaftswissenschaften und Soziologie. Die Domäne Gesellschaft steht für den historisch-gesellschaftlichen Weltzugang. Im engeren Sinne repräsentiert sie die Perspektiven der sozialen Ordnung, der sozialen Organisation und der sozialen Einbettung individuellen und kollektiven menschlichen Denkens und Handelns.

Der sozialwissenschaftliche Zugang zur Domäne Gesellschaft zeichnet sich dadurch aus, dass er den Fokus vor allem auf kollektive politische, wirtschaftliche und soziale Phänomene und Probleme sowie auf gesellschaftlich bedingte individuelle Phänomene und Probleme richtet, insofern gehört auch die Mikroebene dazu. Sozioökonomische Bildung fördert dann „Fähigkeiten und Fertigkeiten in sozial, politisch und ökonomisch geprägten Situationen und Strukturen des gesellschaftlichen Zusammenlebens“ (Weber, 2010, S. 108, Hervorh. RH).

Die Sozioökonomiedidaktik akzeptiert ein verteiltes Definitionsrecht über die Domäne Gesellschaft und das ihr zugeordnete Wissen und Können. Denn nicht nur die wissenschaftlichen Fachdidaktiken und die Allgemeindidaktik, sondern auch Bildungspolitik, Bildungsadministrationen und Bildungspraxis vertreten legitime Vorstellungen über die Strukturierung von schulischen Domänen und ihren Subdomänen, die auch traditionsbildend wirken.

Im Übrigen wendet die Sozioökonomiedidaktik das Prinzip des Pluralismus reflexiv auf sich selbst an. Sie geht davon aus, dass es eine Mehrzahl begründbarer Alternativen für die Strukturierung von Domänen und die Abgrenzung von Fächern gibt. Sie stellt fest, dass es in Europa eine große Vielfalt an Fächerstrukturen und Subdomänenkombinationen gibt, in denen sozioökonomische Bildung verankert ist. Mit Blick auf die unterschiedlichen Bildungstraditionen, Schulstrukturen und Wirtschaftskulturen wäre es sozialwissenschaftlich naiv anzunehmen, man kenne die eine und einzige für die Domänenfrage „effiziente“ Lösung: das Separatfach Wirtschaft.

Genau dies war aber die zentrale Argumentationsfigur, mit der 2010 der sozioökonomische bildungspolitische Konsens aus dem Jahr 2000 öffentlich zur Disposition gestellt wurde. Und genau im Augenblick dieses Angriffes geriet die sozioökonomische Bildung und ihre Didaktik als konzeptionelle Alternative erneut in den Fokus der wissenschaftlichen und öffentlichen Aufmerksamkeit.

Was unterscheidet sozioökonomische Bildung von ihrem ökonomistischen Gegenpol? Was kann man sowohl mit Blick auf ihre Tradition als auch unter Berücksichtigung des aktuellen Diskussionsstandes als ihre Kernmerkmale auszeichnen?

\section{Was unterscheidet die sozioökonomische Bildung?}

Ruft man sich Debatten seit den 1970er Jahren in Erinnerung, kann man die sozioökonomische von der ökonomisch-wirtschaftswissenschaftlichen Bildung anhand von sechs konzeptionellen Charakteristika unterscheiden: 


\section{Sozioökonomische Bildung}

- Bildungs- und Problemorientierung als Leitkriterium für die Auswahl von Wissenschaftswissen aus den Sozialwissenschaften und aus ihren Disziplinen;

- sozialwissenschaftliche Interdisziplinarität als Strukturprinzip von Lehrerausbildung, Fachdidaktik, Curricula, Fach und Unterricht (Wissenschaftsorientierung, Sozialwissenschaftlichkeit);

- sozialwissenschaftlicher und politischer Pluralismus als inhaltlichcurricular-unterrichtliches Prinzip (Kontroversität, Multiparadigmatizität als Form von Wissenschaftsorientierung);

- kritisch-distanzierter und reflektierter Zugang zu wirtschaftlichen Phänomenen, Institutionen und Interessenlagen und analytisch-skeptischer Habitus als Bildungsziel (Kritik);

- gesellschaftliche Kontextualisierung als ein universales Grundphänomen von Wirtschaft und Wirtschaften sowie als eine Grundtatsache der wirtschaftlichen Alltagswelten und des Alltagshandelns der Lernenden (Einbettung);

- multimotivische, mit pluralen und kontroversen wirtschaftlichen Konventionen, Denkmustern, Wertmaßstäben und unterschiedlichen Wissensformen umgehende und im Allgemeinen alltagskompetente Akteure (Subjektorientierung, Wertevielfalt und Wissenspluralität).

Angesichts der aktuellen Auseinandersetzungen um die mit diesen Leitideen umschriebene sozioökonomische Tradition macht es Sinn, die Konturen sozioökonomischer Bildung durch einen Vergleich mit ihrem Gegenpol zu beschreiben (vgl. Tabelle 2). In dieser prononcierten und vereinfachenden Form kann die Gegenüberstellung helfen, die grundsätzlichen fachwissenschaftlichen und fachdidaktischen Alternativen aufzuzeigen, um die sich die Debatte im Kern dreht. Von den in der Übersicht aufgelisteten zwölf Unterscheidungskriterien wurden Bildungsgegenstand, Strukturprinzipien, Wissenschaftsbezug und Disziplinarität sozioökonomischer Bildung oben erläutert; die weiteren Kriterien beschreiben z. B. (Fischer \& Zurstrassen, 2014) und (Hedtke, 2015c).

Nimmt man den Anspruch auf Bildung im beschriebenen Sinne ernst, dann erweist sich die Leistungsfähigkeit sozioökonomischer Bildung vor allem in der Konzipierung und Konkretisierung der Kriterien Bildungsziel, Weltbild, Akteur, Wissensformen und Kritik. Das Kriterium Kritik bezeichnet einen grundsätzlichen Gegensatz der Sozioökonomiedidaktik zum wirtschaftsdidaktischen Mainstream, insofern dieser funktionalistische Ansätze bevorzugt und Employability-, Unternehmer-, Konsum-, Finanz- und Wirtschaftsordnungserziehung weitgehend unkritisch konzipiert. 


\section{Sozioökonomische Bildung}

Tab. 2: Sozioökonomische und ökonomistische Bildung im stilisierten Vergleich

\begin{tabular}{|c|c|c|}
\hline $\begin{array}{ll} & \text { Pole: } \\
\text { Kriterien: } & \end{array}$ & \multicolumn{2}{|c|}{$\leftarrow$ Kontinuum $\rightarrow$} \\
\hline Bildungsgegenstand & $\begin{array}{l}\text { Wirtschaft und Wirtschaften in } \\
\text { der Gesellschaft }\end{array}$ & $\begin{array}{l}\text { „Erkenntnisperspektive“ der } \\
\text { Wirtschaftswissenschaften }\end{array}$ \\
\hline Bildungsziel & $\begin{array}{l}\text { Kontextualisierung von Denken } \\
\text { und Handeln in der / über die } \\
\text { Wirtschaft } \\
\text { Sinnbildung, Reflexion }\end{array}$ & $\begin{array}{r}\text { Ökonomisierung von } \\
\text { wirtschaftlichem Denken und } \\
\text { Handeln } \\
\text { Knappheitsreduktion, } \\
\text { Effizienzsteigerung }\end{array}$ \\
\hline Strukturprinzipien & $\begin{array}{l}\text { Subjektorientierung, } \\
\text { Problemorientierung, } \\
\text { Wissenschaftsorientierung }\end{array}$ & Disziplinorientierung \\
\hline Wissenschaftsbezug & $\begin{array}{l}\text { Sozialwissenschaften: } \\
\text { Wissenschaftswissen über } \\
\text { Wirtschaft }\end{array}$ & $\begin{array}{r}\text { Wirtschaftswissenschaften: } \\
\text { VWL, BWL }\end{array}$ \\
\hline Disziplinarität & $\begin{array}{l}\text { transdisziplinär, multidisziplinär: } \\
\text { sozialwissenschaftliches Feld }\end{array}$ & $\begin{array}{r}\text { monodisziplinär: } \\
\text { Disziplin „Ökonomie“ }\end{array}$ \\
\hline Paradigmatizität & $\begin{array}{l}\text { multiparadigmatisch } \\
\text { Paradigmen aus den } \\
\text { Sozialwissenschaften }\end{array}$ & $\begin{array}{r}\text { monoparadigmatisch } \\
\text { Paradigma der „Ökonomik“ }\end{array}$ \\
\hline Weltbild & kulturalistisch & mechanistisch \\
\hline Rationalität & $\begin{array}{l}\text { Rationalitäten als kulturell } \\
\text { konstituierte, lokale Konstrukte }\end{array}$ & $\begin{array}{l}\text { Zweckrationalität als natürlicher, } \\
\text { objektiver, universaler Fakt }\end{array}$ \\
\hline Akteur & $\begin{array}{l}\text { interpretationsbedürftig; } \\
\text { Handeln als kulturell geprägt, } \\
\text { sozial eingebettet, individuell } \\
\text { und kollektiv sinnhaft konstruiert }\end{array}$ & $\begin{array}{r}\text { kalkulationsbedürftig; } \\
\text { Handeln als individuelle } \\
\text { zweckrationale Reaktion auf } \\
\text { externe Anreize }\end{array}$ \\
\hline Kompetenz & $\begin{array}{l}\text { Ausgangsannahme für Bildung: } \\
\text { wirtschaftlich (alltags-) } \\
\text { kompetente Akteure }\end{array}$ & $\begin{array}{l}\text { Begründung für Bildung: } \\
\text { Akteure mit defizitären } \\
\text { Wirtschaftskompetenzen }\end{array}$ \\
\hline Wissensformen & $\begin{array}{l}\text { subjektives, kulturelles, } \\
\text { pragmatisches und (sozial-) } \\
\text { wissenschaftliches Wissen: } \\
\text { Deutungs- und Handlungswissen }\end{array}$ & $\begin{array}{r}\text { (wirtschaftswissenschaftliches) } \\
\text { Wissenschaftswissen, Begriffs- } \\
\text { und Modellwissen: } \\
\text { deduziertes Anleitungswissen }\end{array}$ \\
\hline Kritik & $\begin{array}{l}\text { Skepsis und Kritik als } \\
\text { wissenschaftsorientierter und } \\
\text { interessenbezogener Habitus }\end{array}$ & k. A. \\
\hline
\end{tabular}

Tabelle erweitert in Anlehnung an (Hedtke, 2015c). 


\section{Sozioökonomische Bildung}

Im Zusammenhang des konzeptionellen Vergleichs muss man betonen, dass es hier um sozialwissenschaftlich begründete Unterscheidungen auf der konzeptionellen Ebene der Sozioökonomiedidaktik geht und nicht um eine Lerninhaltsliste. Auf der materialen Ebene des kollektiv-individuellen Lernens und seiner Regulierung, also in sozioökonomischen Bildungsstandards, Lehrplänen, Lehr-Lern-Materialien, konkreten Unterrichtsreihen und Aufgabenstellungen, gewinnen diese sozioökonomischen Zugriffe auf Wirtschaft gegenüber den exklusiv wirtschaftswissenschaftlichen zwar stark an Relevanz und Gewicht. Während aber die ökonomistischen Bildungskonzepte die Konzepte der anderen Sozialwissenschaften curricular und unterrichtlich ignorieren, verdrängt die Sozioökonomiedidaktik die konventionellen wirtschaftswissenschaftlichen Konzepte keineswegs. Vielmehr sichert sie ihnen sowohl als prinzipielle Alternative zu sozioökonomischen Zugängen und als auch in Form von konkreten alternativen Konzepten einen systematischen Platz in der pluralistisch strukturierten Subdomäne Wirtschaft.

\section{Ausblick}

Sozioökonomiedidaktik und sozioökonomische Bildung sehen sich auf konzeptioneller, bildungspolitischer und curricularer Ebene ähnlichen Verdrängungsversuchen von Seiten ökonomistischer Positionen ausgesetzt wie die sozialwissenschaftliche Tradition der Betriebswirtschaftslehre oder die unorthodoxen Traditionen der Volkswirtschaftslehre (vgl. z. B. Schanz, 2014, Jens \& Rohmahn, 2010, Hedtke, 2015b). Letztlich handelt es sich hier um einen fünffachen Grundsatzkonflikt, der gleichermaßen im Wissenschafts- wie im Bildungssystem ausgefochten wird:

- Soll man einer einzelnen Disziplin einen mehr oder weniger starken Alleinvertretungsanspruch für die Beschreibung und Erklärung wirtschaftlicher Phänomene einräumen (Deutungsmacht)?

- Soll man das „ökonomische“ Paradigma trotz des paradigmatischen Pluralismus in den Sozialwissenschaften in Studium und Unterricht privilegieren (Monoparadigmatizität)?

- Darf man innerhalb der Wirtschaftswissenschaften eine bestimmte theoretisch-politische Strömung zu Lasten anderer Strömungen hervorheben (Parteilichkeit)? ${ }^{3}$

- Kann man auf praktisches wirtschaftliches Denken und Handeln angemessen vorbereiten, wenn man systematisch von sozialer Einbettung, pluralen Praxen und kontroversen Wertpositionen absieht (Handlungsorientierung)? 


\section{Sozioökonomische Bildung}

- Darf man im Pflichtschulsystem Individualismus, Zweckrationalität, monetäre Effizienz und Wachstum als implizite Wertvorstellungen des „ökonomischen“ Paradigmas gegenüber anderen Werten bevorzugen (Normativität)?

Die Sozioökonomiedidaktik beantwortet alle Fragen eindeutig mit Nein. Sie geht davon aus, dass eine Mehrzahl vernünftiger wissenschaftlicher und politischer Perspektiven auf Wirtschaft nicht nur möglich, sondern nötig sind (Harvey, 2015, S. 150). Das gilt aus sozioökonomischer Perspektive für theoretische und praktische Elemente von Bildung und Ausbildung in Schulen und Hochschulen gleichermaßen. Das gilt insbesondere dann, wenn es um die Lösung praktischer individueller oder kollektiver Probleme geht.

In Hochschulen und Schulen gerät die Freiheit des Denkens allerdings unter Druck. Dagegen wehrt sich eine Reihe von kritischen Bewegungen in den Wirtschaftswissenschaften, angetrieben vom Interesse an wissenschaftsbasierter Weltoffenheit, theoretischem Pluralismus und von der Erkenntnis der Pluralität der realen Wirtschaftswelten. Im Schulbereich setzt sich die Sozioökonomiedidaktik dafür ein, dass Schülerinnen und Schüler systematisch angeleitet multiperspektivisch sozialwissenschaftlich denken lernen. Auch in pluralen Welten, mit pluralen Identitäten und pluralen Wertvorstellungen leben die Lernenden heute sowieso. In den Sozialwissenschaften herrscht ein struktureller Pluralismus, ein wissenschaftsorientierter Unterricht über Wirtschaft kann also nur ein pluralistischer sein. Alles andere fiele hinter den Stand der Wissenschaft zurück.

\section{Anmerkungen}

Ich verwende für diesen Text auch Überlegungen und Formulierungen aus Hedtke (2015c).

1 Quellen: Gemeinsame Initiative von Eltern, Lehrern, Wissenschaft, Arbeitgebern und Gewerkschaften, 2000, Retzmann, Seeber, Remmele, \& Jongebloed, 2010, S. 16 f., 34, 71, 81, 114, 117 f., 131.

2 Thomas Retzmann, Günther Seeber, Bernd Remmele, Hans-Carl Jongebloed. Tabelle in Anlehnung an Hedtke, 2015c.

3 In der Regel bevorzugt die konventionelle Wirtschaftsdidaktik ordoliberale und ordnungspolitische Positionen der deutschen Tradition. Sie sind im internationalen Kontext der Volkswirtschaftslehre seit jeher Minderheitsmeinungen mit wenig Resonanz. 


\section{Sozioökonomische Bildung}

\section{Literatur}

Bundeszentrale für politische Bildung (Hrsg.). (2014). Themen und Materialien. Ökonomie und Gesellschaft: Zwölf Bausteine für die schulische und außerschulische politische Bildung. Bonn: Bundeszentrale für politische Bildung.

Davis, J. (2014). ,Pluralism‘ In Economics? A Symposium. Review of Political Economy, 26(4), 477-478.

Diaz-Bone, R. (2015). Die „Économie des conventions“: Grundlagen und Perspektiven eines wirtschaftssoziologischen Paradigmas. Wiesbaden: VS Verlag für Sozialwissenschaften.

Eymard-Duvernay, F., Favereau, O., Orléan, A., Salais, R. \& Thévenot, L. (2011). Werte, Koordination und Rationalität: Die Verbindung dreier Themen durch die „Économie des conventions“. In R. Diaz-Bone (Hrsg.), Soziologie der Konventionen. Grundlagen einer pragmatischen Anthropologie (S. 203-230). Frankfurt am Main: Campus.

Fischer, A. \& Zurstrassen, B. (Hrsg.). (2014). Sozioökonomische Bildung. Bonn.

Gemeinsame Initiative von Eltern, Lehrern, Wissenschaft, Arbeitgebern und Gewerkschaften. (2000). Memorandum: Wirtschaft - notwendig für schulische Allgemeinbildung. Berlin.

[www.sowi-onli-ne.de/reader/oekonomische_politische_bildung/

bda_dgb_u_wirtschaft_notwendig_schulische_allgemeinbildung_2000.html].

Harvey, J. T. (2015). Contending perspectives in economics: A guide to contemporary schools of thought. Cheltenham: Edward Elgar.

Hedtke, R. (2012). Wirtschaftswissenschaft als Politik? Haushalt in Bildung \& Forschung, 1(2), 73-85.

Hedtke, R. (2015a). Mein Wohl als Gemeinwohl: Lobbyismus in der ökonomischen Bildung. In M. Spieker (Hrsg.), Ökonomische Bildung. Zwischen Pluralismus und Lobbyismus (S. 127-172). Schwalbach am Taunus: Wochenschau Verlag.

Hedtke, R. (Hrsg.). (2015b). Was ist und wozu Sozioökonomie? Wiesbaden: Springer Fachmedien.

Hedtke, R. (2015c). Wirtschaft und Gesellschaft. Sozioökonomische Bildung als Innovation durch Tradition. $G W$-Unterricht, (139), i. E.

Jens, U. \& Rohmahn, H. (Hrsg.). (2010). Methodenpluralismus in den Wirtschaftswissenschaften. Marburg: Metropolis Verlag.

Kahsnitz, D. (1999). Sozioökonomische Bildung - ein Kernelement der Allgemeinbildung. Aus Politik und Zeitgeschichte, (35-36), 33-38.

Kokemohr, R. (2007). Bildung als Welt- und Selbstentwurf im Anspruch des Fremden: Eine theoretisch-empirische Annäherung an eine Bildungsprozesstheorie. In H.-C. Koller, W. Marotzki, \& O. Sanders (Hrsg.), Bildungsprozesse und Fremdheitserfahrung. Beiträge zu einer Theorie transformatorischer Bildungsprozesse (S. 13-68). Bielefeld: Transcript-Verlag. 


\section{Sozioökonomische Bildung}

Koller, H.-C. (2007). Probleme einer Theorie transformatorischer Bildungsprozess. In H.-C. Koller, W. Marotzki \& O. Sanders (Hrsg.), Bildungsprozesse und Fremdheitserfahrung. Beiträge zu einer Theorie transformatorischer Bildungsprozesse (S. 69-82). Bielefeld: Transcript-Verlag.

Kornmesser, S., \& Schurz, G. (Hrsg.). (2014). Die multiparadigmatische Struktur der Wissenschaften: Koexistenz, Komplementarität und (In)Kommensurabilität. Wiesbaden: Springer Fachmedien.

Retzmann, T., Seeber, G., Remmele, B. \& Jongebloed, H.-C. (2010). Ökonomische Bildung an allgemeinbildenden Schulen. Bildungsstandards, Standards für die Lehrerbildung: Studie im Auftrag des Gemeinschaftsausschusses der deutschen gewerblichen Wirtschaft. o. O. [Berlin].

Schanz, G. (2014). Eine kurze Geschichte der Betriebswirtschaftslehre. Konstanz: UVK und UTB.

Seeber, G. (1997). Moderne Sozioökonomie als Herausforderung für die ökonomische Bildung. In K.-P. Kruber (Hrsg.), Konzeptionelle Ansätze ökonomischer Bildung (S. 187-210). Bergisch Gladbach: Hobein.

Steinmann, B. (1997). Das Konzept „Qualifizierung für Lebenssituationen“ im Rahmen der ökonomischen Bildung heute. In K.-P. Kruber (Hrsg.), Konzeptionelle Ansätze ökonomischer Bildung (S. 1-22). Bergisch Gladbach: Hobein.

Thévenot, L. (2011). Pluralität kognitiver Formate und Engagements im Bereich zwischen dem Vertrauten und dem Öffentlichen. In R. Diaz-Bone (Hrsg.), Soziologie der Konventionen. Grundlagen einer pragmatischen Anthropologie (S. 255-274). Frankfurt am Main: Campus.

van der Meer-Kooistra, J. \& Vosselman, E. (2012). Research paradigms, theoretical pluralism and the practical relevance of management accounting knowledge. Qualitative Research in Accounting \& Management, 9(3), 245-264.

Weber, B. (2010). Weder isolierte ökonomische, noch dominante politische Bildung! Sozialwissenschaftliche Bildung als Ausweg? Seminar - Lehrerbildung und Schule, 16(2), 104-111.

\section{Verfasser}

Prof. Dr. Reinhold Hedtke

Universität Bielefeld, Fakultät für Soziologie

Postfach 100131

D-33501 Bielefeld

E-Mail: reinhold.hedtke@uni-bielefeld.de

Internet: www.uni-bielefeld.de/soz/ag/hedtke; www.iboeb.org 